\title{
First ${ }^{80} \mathrm{Se}(\mathbf{n}, \gamma)$ cross section measurement with high reso- lution in the full stellar energy range $1 \mathrm{eV}-100 \mathrm{keV}$ and its astrophysical implications for the $s$-process
}

V. Babiano-Suarez ${ }^{1}$, J. Balibrea-Correa ${ }^{1}$, L. Caballero-Ontanaya ${ }^{1}$, C. Domingo-Pardo ${ }^{1}$, I. Ladarescu ${ }^{1}$, J. Lerendegui-Marco ${ }^{2}$, J. L. Tain ${ }^{1}$, F. Calviño ${ }^{18}$, A. Casanovas ${ }^{18}$, A. TarifeñoSaldivia $^{18}$, C. Guerrero ${ }^{2}$, O. Aberle ${ }^{3}$, V. Alcayne ${ }^{4}$, S. Amaducci ${ }^{5,6}$, J. Andrzejewski ${ }^{7}$, L. Audouin ${ }^{8}$, M. Bacak ${ }^{3,9,10}$, M. Barbagallo ${ }^{3,11}$, S. Bennett ${ }^{12}$, E. Berthoumieux ${ }^{10}$, J. Billowes ${ }^{12}$, D. Bosnar ${ }^{13}$, A. Brown ${ }^{14}$, M. Busso ${ }^{15,16}$, M. Caamaño ${ }^{17}$, M. Calviani ${ }^{3}$, D. Cano-Ott ${ }^{4}$, F. Cerutti ${ }^{3}$, E. Chiaveri ${ }^{3,12}$, N. Colonna ${ }^{11}$, G. Cortés ${ }^{18}$, M. A. CortésGiraldo $^{2}$, L. Cosentino ${ }^{5}$, S. Cristallo ${ }^{15,19}$, L. A. Damone ${ }^{11,20}$, P. J. Davies ${ }^{12}$, M. Diakaki ${ }^{21,3}$, M. Dietz ${ }^{22}$, R. Dressler ${ }^{23}$, Q. Ducasse ${ }^{24}$, E. Dupont ${ }^{10}$, I. Durán ${ }^{17}$, Z. Eleme ${ }^{25}$, B. FernándezDomínguez $^{17}$, A. Ferrari ${ }^{3}$, P. Finocchiaro ${ }^{5}$, V. Furman ${ }^{26}$, K. Göbel ${ }^{27}$, R. Garg ${ }^{22}$, A. Gawlik-Ramięga ${ }^{7}$, S. Gilardoni ${ }^{3}$, I. F. Gonçalves ${ }^{28}$, E. González-Romero ${ }^{4}$, F. Gunsing ${ }^{10}$, H. Harada ${ }^{29}$, S. Heinitz ${ }^{23}$, J. Heyse ${ }^{30}$, D. G. Jenkins ${ }^{14}$, A. Junghans ${ }^{31}$, F. Käppeler ${ }^{32}$, Y. $\mathrm{Kadi}^{3}$, A. Kimura ${ }^{29}$, I. Knapová ${ }^{33}$, M. Kokkoris ${ }^{21}$, Y. Kopatch ${ }^{26}$, M. Krtička ${ }^{33}$, D. Kurtulgil ${ }^{27}$, C. Lederer-Woods ${ }^{22}$, H. Leeb ${ }^{9}$, S. J. Lonsdale ${ }^{22}$, D. Macina ${ }^{3}$, A. Manna ${ }^{34,35}$, T. Martínez ${ }^{4}$, A. Masi ${ }^{3}$, C. Massimi ${ }^{34,35}$, P. Mastinu ${ }^{36}$, M. Mastromarco ${ }^{3}$, E. A. Maugeri ${ }^{23}$, A. Mazzone ${ }^{11,37}$, E. Mendoza ${ }^{4}$, A. Mengoni ${ }^{38}$, V. Michalopoulou ${ }^{21,3}$, P. M. Milazzo ${ }^{39}$, F. Mingrone ${ }^{3}$, J. Moreno-Soto ${ }^{10}$, A. Musumarra ${ }^{5,40}$, A. Negret ${ }^{41}$, R. Nolte ${ }^{24}$, F. Ogállar ${ }^{42}$, A. Oprea $^{41}$, N. Patronis ${ }^{25}$, A. Pavlik ${ }^{43}$, J. Perkowski ${ }^{7}$, L. Persanti ${ }^{11,15,19}$, C. Petrone ${ }^{41}$, E. Pirovano ${ }^{24}$, I. Porras ${ }^{42}$, J. Praena ${ }^{42}$, J. M. Quesada ${ }^{2}$, D. Ramos-Doval ${ }^{8}$, T. Rauscher ${ }^{44,45}$, R. Reifarth ${ }^{27}$, D. Rochman ${ }^{23}$, Y. Romanets ${ }^{28}$, C. Rubbia ${ }^{3}$, M. Sabaté-Gilarte ${ }^{2,3}$, A. Saxena ${ }^{46}$, P. Schillebeeckx ${ }^{30}$, D. Schumann ${ }^{23}$, A. Sekhar ${ }^{12}$, A. G. Smith ${ }^{12}$, N. V. Sosnin ${ }^{12}$, P. Sprung ${ }^{23}$, A. Stamatopoulos ${ }^{21}$, G. Tagliente ${ }^{11}$, L. Tassan-Got ${ }^{3,21,8}$, Th. Thomas ${ }^{27}$, P. Torres-Sánchez ${ }^{42}$, A. Tsinganis ${ }^{3}$, J. Ulrich ${ }^{23}$, S. Urlass ${ }^{31,3}$, S. Valenta ${ }^{33}$, G. Vannini ${ }^{34,35}$, V. Variale ${ }^{11}$, P. Vaz $^{28}$, A. Ventura ${ }^{34}$, D. Vescovi ${ }^{15}$, V. Vlachoudis ${ }^{3}$, R. Vlastou ${ }^{21}$, A. Wallner ${ }^{47}$, P. J. Woods ${ }^{22}$, T. Wright ${ }^{12}$, and P. Žugec ${ }^{13}$ and the n_TOF Collaboration

\footnotetext{
${ }^{1}$ Instituto de Física Corpuscular, CSIC - Universidad de Valencia, Spain

${ }^{2}$ Universidad de Sevilla, Spain

${ }^{3}$ European Organization for Nuclear Research (CERN), Switzerland

${ }^{4}$ Centro de Investigaciones Energéticas Medioambientales y Tecnológicas (CIEMAT), Spain

${ }^{5}$ INFN Laboratori Nazionali del Sud, Catania, Italy

${ }^{6}$ Dipartimento di Fisica e Astronomia, Università di Catania, Italy

${ }^{7}$ University of Lodz, Poland

${ }^{8}$ Institut de Physique Nucléaire, CNRS-IN2P3, Univ. Paris-Sud, Université Paris-Saclay, F-91406 Orsay Cedex, France

${ }^{9}$ TU Wien, Atominstitut, Stadionallee 2, 1020 Wien, Austria

${ }^{10}$ CEA Irfu, Université Paris-Saclay, F-91191 Gif-sur-Yvette, France

${ }^{11}$ Istituto Nazionale di Fisica Nucleare, Sezione di Bari, Italy

${ }^{12}$ University of Manchester, United Kingdom

${ }^{13}$ Department of Physics, Faculty of Science, University of Zagreb, Zagreb, Croatia
} 


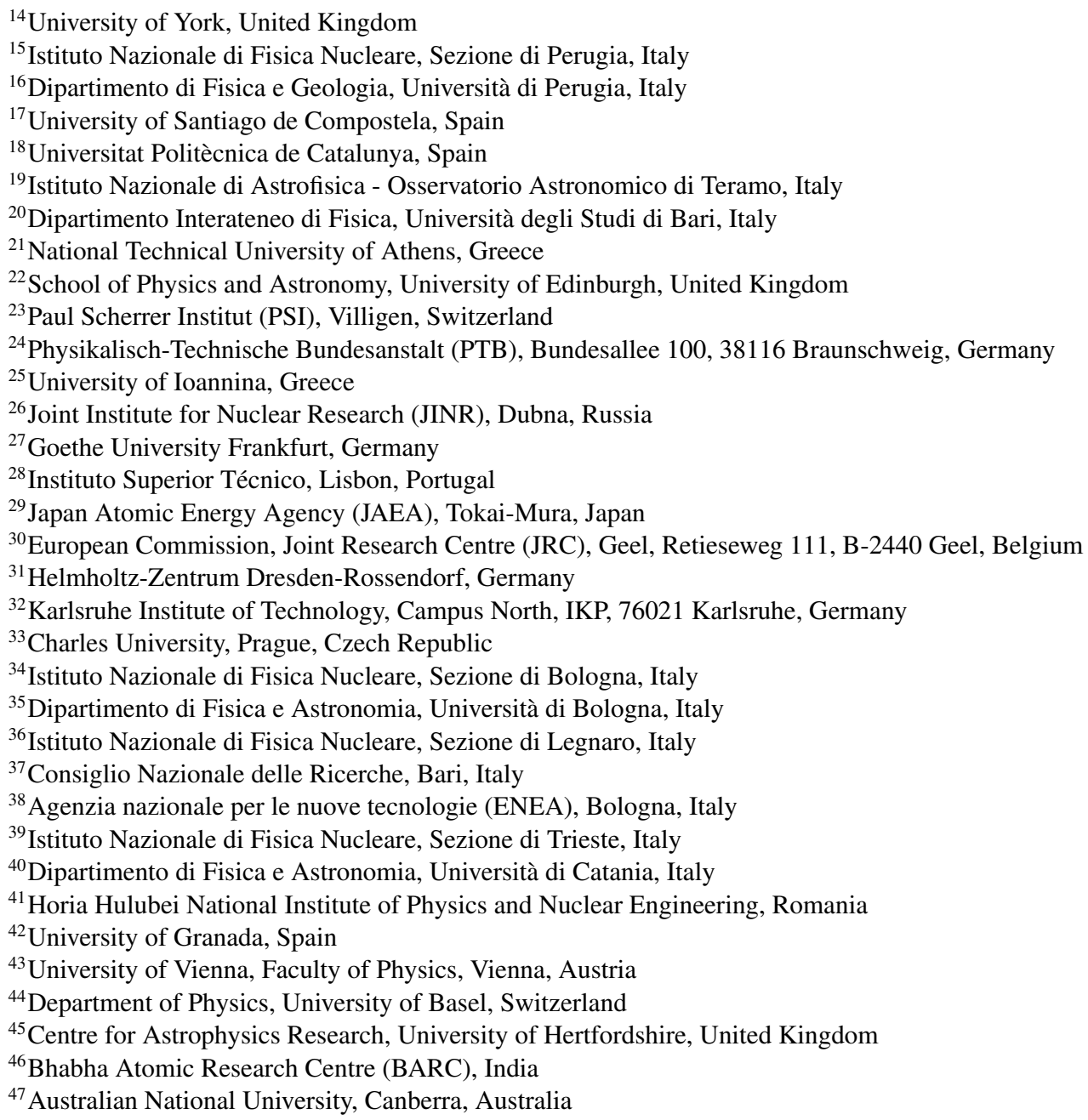

Abstract. Most elements heavier than iron have been generated in the stellar media by means of neutron capture reactions, approximately half are produced by the slow neutron capture or $s$-process. Radiative neutron capture cross section measurements are of fundamental importance for the study of this mechanism. In this contribution we present a brief summary on the measurement and results for the ${ }^{80} \mathrm{Se}(\mathrm{n}, \gamma)$ cross-section. The experiment was carried out at CERN n_TOF EAR1 via the time of flight (ToF) technique, using four $\mathrm{C}_{6} \mathrm{D}_{6}$ scintillation detectors with very fast response. More than a hundred new resonances have been analyzed for the first time with a high accuracy. The MACS obtained at $\mathrm{kT}=8 \mathrm{keV}$ is $36 \%$ smaller than the recommended value in KADo$\mathrm{NiS}$. Some of the astrophysical implications of this result are elucidated in this contribution.

\section{Introduction}

The $s$-process is one of the main nucleosynthesis mechanisms proposed to explain the abundance curve of elements [1]. For the nucleosynthesis of elements with mass $A>90$, the 
$s$-process is ascribed to low mass stars during their AGB evolutionary phase, in which neutrons are released by the reactions ${ }^{22} \mathrm{Ne}(\alpha, \mathrm{n}){ }^{25} \mathrm{Mg}$ and ${ }^{13} \mathrm{C}(\alpha, \mathrm{n}){ }^{16} \mathrm{O}$ corresponding to He-flash and ${ }^{13} \mathrm{C}$-pocket, respectively [2]. For the mass region $60<A<90$ the weak $s$-process was introduced [1]. This additional component takes place in massive stars where neutrons are released by the reactions ${ }^{22} \mathrm{Ne}(\alpha, \mathrm{n}){ }^{25} \mathrm{Mg}$ and ${ }^{12} \mathrm{C}\left({ }^{12} \mathrm{C}, \mathrm{n}\right){ }^{23} \mathrm{Mg}$ during the core-He and shell-C burning stages [2]. For both main and weak $s$-process components the relatively low neutron flux allows unstable nuclei produced to $\beta$-decay before capturing another neutron. In some specific cases where the beta-decay half-life spans between weeks and several years, the unstable nucleus can capture another neutron before decaying, inducing a split in the $s$-process path. In these branching-point nuclei temperature and neutron density play an important role in shaping the final isotopic-abundance pattern. Because of the branching sensitivity to the varying physical conditions and evolutionary time-scales, stellar models [3-7] are required for a realistic assessment of the $s$-process production yields, based on the neutron capture cross sections and $\beta$-decay rates of all isotopes involved.

${ }^{79} \mathrm{Se}\left(3.27 \times 10^{5} \mathrm{y}[8]\right)$ is one of the most relevant $s$-process branching [9] isotopes, for it allows to constrain the thermal conditions during the last evolutionary stage of massive-stars, before they explode as supernova. The thermal sensitivity of the nucleosynthesis around ${ }^{79} \mathrm{Se}$ arises from a few quantum levels at low excitation energy that are populated in the stellar media. The comparison between the abundances observed for the $s$-only isotopes of $\mathrm{Kr}$ [10], and those predicted by the stellar models, will give information about the thermal conditions of the stellar media. To this aim one needs to know both, the cross-section of the branching nucleus ${ }^{79} \mathrm{Se}$, whose measurement is scheduled for 2022 [11], and also the cross sections of the neighbouring stable nuclei. In this context, we carried out the neutron capture cross section measurement of the ${ }^{80} \mathrm{Se}$ isotope using the ToF technique, at CERN n_TOF [12], under the framework of the ERC-funded project HYMNS [13]. Experimentally, there existed only one previous measurement on ${ }^{80} \mathrm{Se}$ using the ToF technique [14]. However, the latter had a poor neutron-energy resolution and a rather limited neutron energy range. These drawbacks have been remarkably improved with the present high resolution measurement, which covers the entire energy range of astrophysical interest between $1 \mathrm{eV}$ and $100 \mathrm{keV}$.

\section{Experimental setup and measuring technique}

At CERN n_TOF [15] neutrons are produced via spallation reactions of a proton beam impinging on a lead block. $7 \times 10^{12}$ protons reach the spallation target every proton-pulse, thereby generating $\sim 300$ neutrons per incident proton. The outcoming neutrons pass through a layer of borated water which moderates their energy leading to a white neutron flux covering the range from $\mathrm{meV}$ to $\mathrm{GeV}$ in each single shot. Charged particles are removed from the beam using a magnet, whereas the rest of particles are collimated into a beam by two different collimators along the beam line. Neutrons travel $\sim 185 \mathrm{~m}$ to reach the experimental area EAR1, where the pulsed beam shows a Gaussian profile of $18 \mathrm{~mm}$ FwHM at the sample position [16]. In addition to neutrons, a flux of $\gamma$-rays generated by spallation- and neutron captures in the moderator, reaches the experimental hall. The prompt $\gamma$-rays is called $\gamma$-flash and saturates the response of all radiation detectors for few $\mu$ s with a very steep rise-edge. This is utilized to determine the time reference for determining the ToF of each neutron bunch. Therefore, the kinetic energy in $\mathrm{eV}$ of a neutron with a measured $\mathrm{ToF} t_{T o F}(\mu \mathrm{s})$ is given by Eq. 1 , in which $L$ is the flight distance in $\mathrm{m}$. The short bunch width of $6 \mathrm{~ns}$ RMs and the long flight distance ensure an excellent neutron-energy resolution.

$$
E_{n}=\left(\frac{72.2977 L}{t_{T o F}}\right)^{2}
$$


Table 1. Disk shaped samples of $20 \mathrm{~mm}$ diameter used during the ${ }^{80} \mathrm{Se}(\mathrm{n}, \gamma) \mathrm{ToF}$ experiment. Dummy is not included.

\begin{tabular}{cccc}
\hline Sample & Pureness (\%) & Mass $(\mathrm{g})$ & Thickness $(\mathrm{mm})$ \\
\hline${ }^{80} \mathrm{Se}$ & $99.87(10)$ & $2.965(5)$ & $3.50(1)$ \\
${ }^{197} \mathrm{Au}$ & 99.99 & $0.600(5)$ & $0.10(1)$ \\
${ }^{n a t} \mathrm{~Pb}$ & 100 & $7.300(5)$ & $2.10(1)$ \\
\hline
\end{tabular}

Fig. 1 shows a photograph of the experimental setup consisting in four $\mathrm{C}_{6} \mathrm{D}_{6}$ scintillation detectors [17] placed at $125^{\circ}$ with respect to the beam line, and their faces pointing at the capture sample from $\sim 10 \mathrm{~cm}$ distance. These detectors are built in carbon fiber to minimize contaminant neutron captures in structural materials, an effect known as intrinsic neutron sensitivity. Each detector contains approximately 11 volume of $\mathrm{C}_{6} \mathrm{D}_{6}$ scintillation liquid, which is coupled to a traditional PMT to give a very fast response.

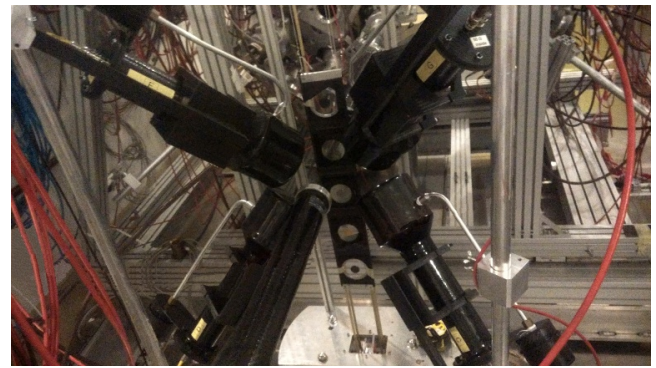

Figure 1. General view of experimental setup mounted in EAR1 for the measurement of the ${ }^{80} \mathrm{Se}(\mathrm{n}, \gamma)$ cross section.

Their low efficiency allows one to apply the Pulse-Height Weighting Technique [18, 19].

The samples used for this experiment are listed in Tab. 1. Apart from the selenium sample under study, gold was measured to normalize the obtained ${ }^{80} \mathrm{Se}(\mathrm{n}, \gamma)$ yield by means of the Saturated Resonance Method (SRM) [20]. The ancillary lead sample was measured to estimate the background contribution corresponding to neutrons scattered by the sample and captured in the structural material of the experimental hall [21]. In addition, a replica of the ${ }^{80} \mathrm{Se}$ sample without the sample itself, called empty sample, was measured to estimate the background component unrelated to the sample and caused by neutrons captured in the surroundings.

\section{Data analysis}

The measured count-rate as a function of the ToF was transformed into a neutron capture yield by means of Eq. 2. $N^{w}$ stands for the weighted number of counts already background subtracted, the neutron flux is indicated by $N_{n}$ and the energy of the capture cascade corresponding is $E_{C} \approx S_{n}+E_{n}$.

$$
Y\left(E_{n}\right)=\frac{N^{w}\left(E_{n}\right)}{N_{n}\left(E_{n}\right) E_{C}\left(E_{n}\right)}
$$

Before calculating the capture yield, the performance of all $\mathrm{C}_{6} \mathrm{D}_{6}$ detectors along the experiment was studied in terms of gain and count-rate stability to filter-out possible accidental errors or systematic bias in the data taking. The detectors were calibrated using radioactive sources of ${ }^{137} \mathrm{Cs},{ }^{88} \mathrm{Y}$ and $\mathrm{AmBe}$ together with the ${ }^{197} \mathrm{Au}(\mathrm{n}, \gamma)$ deposited energy spectra, ensuring a valid range between $662 \mathrm{keV}$ and $6.512 \mathrm{MeV}$. Additionally, a series of Monte Carlo simulations of the entire experimental setup were performed to calculate the weighting function, which was applied following the methodology described in Ref. [19]. The latter includes yield-correction factors to account for the threshold-effects in the detectors, as well as $\gamma$-ray summing and conversion-electron effects. The neutron flux available during the experiment was determined from the evaluated version measured with high precision at $\mathrm{n} \_$TOF [22]. 
Disagreements between the determined and real neutron flux, or between simulated and true experimental setup were taken into account using the aforementioned SRM [20]. After subtracting all backgrounds present in the measurement [21], the capture yield was obtained using Eq. 2.

Once the yield was calculated, a resonance analysis was carried out using the SAMMY code [23]. This code is based on the R-matrix theory that gives a phenomenological description of the neutron induced reactions [24, 25]. SAMMY performs a Bayesian fit to the experimental data using an initial set of R-matrix resonance parameters and employing, by de-

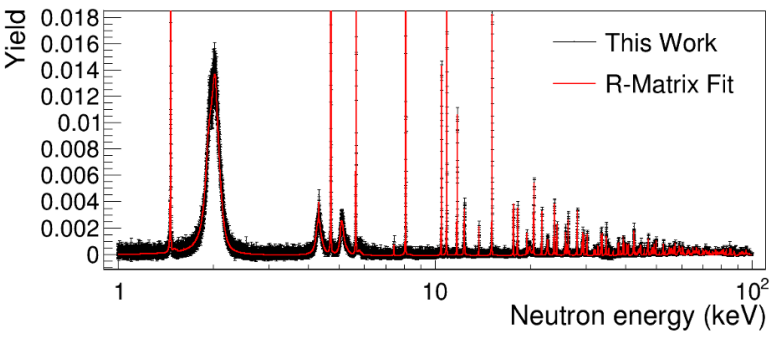

Figure 2. Measured ${ }^{80} \mathrm{Se}(\mathrm{n}, \gamma)$ yield between $1 \mathrm{keV}$ and 100 $\mathrm{keV}$ (red data-points) and R-matrix analysis fit (red curve). fault, the recommended Reich-

Moore approximation. Experimental effects such as Doppler broadening or neutron multiple scattering effects are taken into account in SAMMY by providing experimental data as temperature or sample thickness. Fig. 2 shows the final capture yield obtained in this work and fitted with SAMMY. In summary, 113 resonances have been analyzed, 98 of them for the first time and only 15 were previously known from transmission [26, 27]. There was no resonance data available from the previous ToF measurement [14]. The high resolution and high statistics of the measurement led us to propose a spin-parity change in the first resonance at 1.47 $\mathrm{keV}$, and to individually analyze the multiple scattering component in the second resonance at $1.98 \mathrm{keV}$ of neutron energy.

\section{MACS}

During the $s$-process, neutrons in the stars are thermalized following the Maxwell distribution of velocities. Hence, the MACS is the input quantity required in stellar models to study this nucleosynthesis mechanism. At a temperature $T$, the MACS is given by Eq. 3 .

$$
\operatorname{MACS}=\frac{\left\langle\sigma_{v}\right\rangle}{v_{T}}=\frac{2}{(k T)^{2} \sqrt{\pi}} \int_{E_{i}=0}^{E_{f}=\infty} \sigma_{\gamma}(E) E e^{-E / k T} d E .
$$

The MACS for ${ }^{80} \mathrm{Se}$ was determined from the measured capture yield (Fig. 2) by means of the SAMMY code. Fig. 3 shows the MACS of ${ }^{80} \mathrm{Se}(\mathrm{n}, \gamma)$ determined in this work and compared to that obtained from resonance parameters fom the JEFF3.3 evaluated library [28]. Also the MACS from KADo$\mathrm{NiS}$ [29] and that calculated in the previous measurement [14] are displayed for comparison.

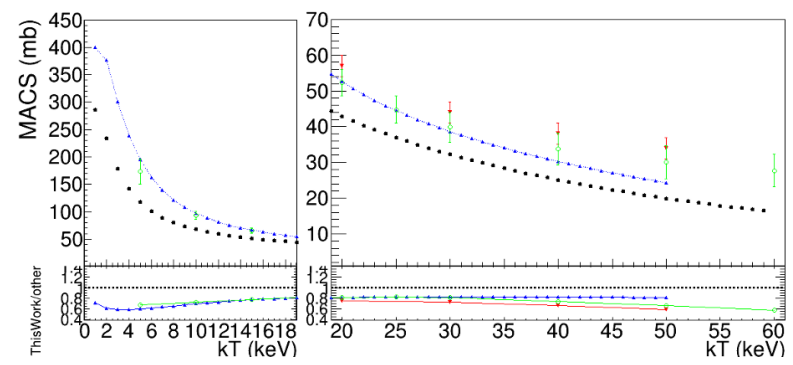

Figure 3. MACS of ${ }^{80} \mathrm{Se}(\mathrm{n}, \gamma)$ determined in this work compared to evaluated libraries and previous measurement. 
The ratio between this work and the rest of contributions is displayed in the bottom panel of the figure. On average, our MACS is between $20 \%$ and $40 \%$ lower than previous values.

Along the TP-AGB evolutionary stage, thermal energies of $k T \sim 8 \mathrm{keV}$ and $k T \sim 23 \mathrm{keV}$ are reached. In massive stars neutrons are produced at energies of $K T \sim 26 \mathrm{keV}$ and $k T \sim 90 \mathrm{keV}$ during core He-burning and shell C-burning, respectively [2]. Differences in MACS at these temperatures are more influential on the astrophysical aspects. Tab. 2 displays the different results at energies close to these points, in which the compared models have available data. At $k T=10 \mathrm{keV}$, the MACS obtained from the JEFF3.3 evaluated library is compatible with that available in KADoNiS, but both are $40 \%$ overestimated compared to the new result at this energy. Relative differences decrease at $30 \mathrm{keV}$, where the new MACS is smaller by $30 \%$. Finally, following this trend, the new value for the MACS at $90 \mathrm{keV}$ is 25\% smaller than that obtained from JEFF3.3. However, the MACS in KADoNiS is overestimated by a factor of 2.5 , whereas no value is available at this point from the previous measurement [14].

Table 2. Value of the MACS at three different temperatures. Only statistical uncertainties are included in these results.

\begin{tabular}{cccc}
\hline & \multicolumn{3}{c}{ MACS $(\mathrm{mb})$} \\
& at $10 \mathrm{keV}$ & at $30 \mathrm{keV}$ & at $90 \mathrm{keV}$ \\
\hline JEFF 3.3 & 96.9 & 38.7 & 11.9 \\
KADoNis & $93.6(6.8)$ & $39.8(4.1)$ & $23.7(4.3)$ \\
Walter et al. & - & $44(3)$ & - \\
\hline This Work & $\mathbf{6 7 . 6 ( 4 )}$ & $\mathbf{3 2 . 2 ( 3 )}$ & $\mathbf{9 . 5 ( 1 )}$ \\
\hline
\end{tabular}

\section{Summary and outlook}

A high resolution measurement of the ${ }^{80} \mathrm{Se}(\mathrm{n}, \gamma)$ cross section has been carried out at CERN $\mathrm{n}$ _TOF covering for the first time a very broad neutron-energy range, from meV up to $100 \mathrm{keV}$. The MACS could be accurately determined for the relevant stellar temperatures, significantly improving previous statistical and systematic uncertainties. As next steps we intend to investigate the impact of the new results on weak s-process nucleosynthesis and massive stars [30]. This results will be required for a full interpretatio of the ${ }^{79} \mathrm{Se}$ branching, whose measurement is scheduled at CERN n_TOF for 2022 [11].

\section{Acknowledgement}

We acknowledge support from from the European Research Council (ERC) under the European Union's Horizon 2020 research and innovation program (grant agreement No. 681740), and the Spanish Science Ministry for funding projects FPA2017-83946-C2-1-P and PID2019104714GB-C21. This work is part of the PhD Thesis of V. Babiano-Suarez.

\section{References}

[1] E.M. Burbidge, G.R. Burbidge, W.A. Fowler, F. Hoyle, Reviews of Modern Physics 29, 547 (1957)

[2] F. Käppeler, Progress in Particle and Nuclear Physics 66, 390 (2011)

[3] S. Degl'Innocenti, P.G. Prada Moroni, M. Marconi, A. Ruoppo, Astrophysics and Space Science 316, 25 (2008) 
[4] B. Paxton, L. Bildsten, A. Dotter, F. Herwig, P. Lesaffre, F. Timmes, Astrophysical Journal Supplement 192, 3 (2011)

[5] M. Pignatari, F. Herwig, R. Hirschi, M. Bennett, G. Rockefeller, C. Fryer, F.X. Timmes, C. Ritter, A. Heger, S. Jones et al., Astrophysical Journal Supplement 225, 24 (2016)

[6] R. Gallino, C. Arlandini, M. Busso, M. Lugaro, C. Travaglio, O. Straniero, A. Chieffi, M. Limongi, The Astrophysical Journal 497, 388 (1998)

[7] S. Cristallo, L. Piersanti, O. Straniero, The FRUITY database on AGB stars: past, present and future, in Journal of Physics Conference Series (2016), Vol. 665 of Journal of Physics Conference Series, p. 012019, 1405 . 3392

[8] G. Jörg, R. Bühnemann, S. Hollas, N. Kivel, K. Kossert, S. Van Winckel, C.L.v. Gostomski, Applied Radiation and Isotopes 68, 2339 (2010)

[9] F. Käppeler, Progress in Particle and Nuclear Physics 43, 419 (1999)

[10] U. Ott, F. Begemann, J. Yang, S. Epstein, Nature 332, 700 (1988)

[11] V. Babiano-Suárez, J. Balibrea-Correa, L. Caballero, F. Calviño, D. Cano-Ott, A. Casanovas, N. Colonna, S. Cristallo, C. Domingo-Pardo, C. Guerrero et al., Tech. Rep. CERN-INTC-2020-065, INTC-P-580, CERN, Geneva (2020), http://cds. cern.ch/record/2731962

[12] V. Babiano-Suárez, L. Caballero, C. Domingo-Pardo, C. Guerrero, A. Tarifeño-Saldivia, Tech. Rep. CERN-INTC-2018-005, INTC-P-536, CERN, Geneva (2018), http:// cds. cern. ch/record/2299660

[13] High-sensitivitY Measurements of key stellar Nucleo-Synthesis reactions (HYMNS), ERC-consolidator grant agreement no. 681740, PI: C. Domingo Pardo.

[14] G. Walter, H. Beer, F. Kaeppeler, G. Reffo, F. Fabbri, Astronomy and Astrophysics 167, $186(1986)$

[15] C. Guerrero, A. Tsinganis, E. Berthoumieux, M. Barbagallo, F. Belloni, F. Gunsing, C. Weiß, E. Chiaveri, M. Calviani, V. Vlachoudis et al., European Physical Journal A 49, 27 (2013)

[16] S. Andriamonje, M. Calviani, Y. Kadi, R. Losito, V. Vlachoudis, E. Berthoumieux, F. Gunsing, Y. Giomataris, T. Papaevangelou, C. Guerrero et al., Journal of Korean Physical Society 59, 1601 (2011)

[17] P.F. Mastinu, R. Baccomi, E. Berthoumieux, D. Cano-Ott, F. Gramegna, C. Guerrero, C. Massimi, P.M. Milazzo, F. Mingrone, J. Praena et al., Tech. Rep. CERN-INTC-2013002, CERN (2013)

[18] R.L. Macklin, J.H. Gibbons, Physical Review 159, 1007 (1967)

[19] U. Abbondanno, G. Aerts, H. Alvarez, S. Andriamonje, A. Angelopoulos, P. Assimakopoulos, C.O. Bacri, G. Badurek, P. Baumann, F. Bečváŕ et al., Nuclear Instruments and Methods in Physics Research A 521, 454 (2004)

[20] R.L. Macklin, J. Halperin, R.R. Winters, Nuclear Instruments and Methods 164, 213 (1979)

[21] P. Žugec, N. Colonna, D. Bosnar, S. Altstadt, J. Andrzejewski, L. Audouin, M. Barbagallo, V. Bécares, F. Bečvář, F. Belloni et al., Nuclear Instruments and Methods in Physics Research A 760, 57 (2014), 1406.6865

[22] M. Barbagallo, C. Guerrero, A. Tsinganis, D. Tarrío, S. Altstadt, S. Andriamonje, J. Andrzejewski, L. Audouin, V. Bécares, F. Bečvář et al., European Physical Journal A 49, 156 (2013)

[23] N.M. Larson, ORNL/TM-9179/R8, Oak Ridge National Laboratory, Oak Ridge, TN (2008)

[24] E.P. Wigner, L. Eisenbud, Physical Review 72, 29 (1947) 
[25] A.M. Lane, R.G. Thomas, Reviews of Modern Physics 30, 257 (1958)

[26] G.M. Novoselov, V.G. Krivenko, L.L. Litvinskij, I.M. Simonov, Jadernye Konstanty (Nuclear Constants) 1-2, 3 (1995)

[27] S.F. Mughabghab, D.I. Garber, Neutron cross sections. Volume I. Resonance parameters (Academic Press Inc, United States, 1973)

[28] A.J.M. Plompen, O. Cabellos, C. De Saint Jean, M. Fleming, A. Algora, M. Angelone, P. Archier, E. Bauge, O. Bersillon, A. Blokhin et al., European Physical Journal A 56, $181(2020)$

[29] I. Dillmann, T. Szücs, R. Plag, Z. Fülöp, F. Käppeler, A. Mengoni, T. Rauscher, Nuclear Data Sheets 120, 171 (2014)

[30] M. Pignatari, R. Gallino, M. Heil, M. Wiescher, F. Käppeler, F. Herwig, S. Bisterzo, The Astrophysical Journal 710, 1557 (2010) 